CASE REPORT

\title{
Rothia mucilaginosa Bacteremia Associated with HIV and Non-Hodgkin's Lymphoma: A Case Report and Brief Review of Literature
}

\author{
Christopher Miller ${ }^{1}$, Carmen Tichindelean ${ }^{2}$, Lisa Blanchette ${ }^{2}$ \\ ${ }^{1}$ Novant Health Presbyterian Medical Center; Department of Pharmacy; Charlotte, NC, USA \\ ${ }^{2}$ Novant Health Infectious Disease Specialists, Charlotte; Charlotte, NC, USA
}

\begin{abstract}
Rothia mucilaginosa is part of the normal flora of the oral cavity and is typically is not associated with acute infection. However, growing evidence suggests that Rothia mucilaginosa can be an opportunistic pathogen in immunocompromised hosts. We present a case of Rothia bacteremia in a patient with a compromised immune system due to human immunodeficiency virus (HIV) and stage IV bulky diffuse large B cell lymphoma affecting the pharyngeal space. J Microbiol Infect Dis 2017; 7(3):148-150
\end{abstract}

Keywords: Rothia mucilaginosa, human immunodeficiency virus, non-Hodgkin's lymphoma, bacteremia, opportunistic infection, case

\section{INTRODUCTION}

Rothia mucilaginosa, previously known as Stomatococcus mucilaginosus, is a grampositive encapsulated, non-spore-forming and non-motile coccus bacterium [1]. A member of the family of Micrococcaceae, it colonizes the human upper respiratory tract and oral cavity as normal host flora [2]. When first discovered, Rothia was considered a commensal, nonpathogenic organism [2]. However, there have been a growing number of invasive infections reported in the literature among immunocompromised hosts including bacteremia, endocarditis, pneumonia, and osteomyelitis [1]. Factors contributing to the risk for invasive disease include hematologic malignancy with severe neutropenia, diabetes, alcoholism, chronic liver disease, and human immunodeficiency virus (HIV). Rothia has been found to be a more prevalent organism in the oral flora of patients with HIV compared with oral flora of non-HIV infected hosts where Rothia comprises a very small proportion of the overall commensal oral organisms [3].

The clinical implication of bacteremia associated with Rothia is currently unknown as it is most frequently interpreted as a blood culture contaminate. In non-immunocompromised hosts where Rothia is identified in a single blood culture, this may be an appropriate conclusion [4]. Treatment of invasive Rothia infections also presents unique challenges as isolates have poor growth in vitro making susceptibility testing difficult [4]. Historically, Rothia spp. has shown susceptibility to most beta-lactam antibiotics, including penicillin. However, penicillin-resistant strains have been identified, with resistance rates as high as $50 \%$ in some reports [4]. Vancomycin and rifampin have demonstrated consistent activity against Rothia while macrolides and aminoglycosides are significantly less effective [5]. We report a case of Rothia bacteremia in a patient with HIV and stage IV diffuse large B cell lymphoma affecting the pharyngeal space.

\section{CASE}

A 49-year-old man was admitted to surgical services with intractable abdominal pain, fever, and leukocytosis. Past medical history was significant for HIV and stage IV bulky diffuse large B cell lymphoma diagnosed six years prior. Previous lymphoma treatment included five cycles of cyclophosphamide, doxorubicin, vincristine, and prednisone. The patient was not 
on highly active antiretroviral therapy (HAART) prior to this hospital admission. A PET scan on admission revealed widespread lymphoma with involvement of the neck, chest, abdomen, and pelvis including a small bowel tumor with intussusception resulting in a small bowel obstruction. Due to this obstruction, surgical intervention with an exploratory laparotomy was performed on hospital day one. On hospital day three, he was found to have new self-limiting hemoptysis and epistaxis. A CT of the neck revealed lymphoma involvement of the nasopharynx and pharynx as the likely cause of hemoptysis and epistaxis.

Also on admission, two sets of blood cultures were obtained given fever and leukocytosis in the setting of untreated HIV. One of two sets revealed gram positive cocci, later identified as Rothia mucilaginosa. Vitek®2 was used for organism identification. Unfortunately, organism susceptibility was unable to be determined due to failure to grow on culture media. Intravenous antibiotic therapy with vancomycin was initiated. Repeat blood cultures were obtained to establish clearance of the organism. The patient became afebrile on day two of antibiotic therapy and leukocytosis normalized on day five of therapy. Intravenous vancomycin therapy was continued for a total of ten days after the first negative blood culture with complete resolution of signs of infection. Further workup, including CD4 count was obtained to guide HIV management. CD4 count was found to be 73 cells $/ \mathrm{mm} 3$ and the patient was initiated on HAART with emtricitabine, tenofovir, and dolutegravir; PCP prophylaxis with sulfamethoxazole/trimethoprim was also initiated. Regular outpatient follow-up for lymphoma and HIV management continued post-discharge with routine monitoring. The patient remained asymptomatic from infection standpoint at 4 and 10 weeks after the vancomycin was discontinued despite ongoing chemotherapy.

\section{DISCUSSION}

Immunocompromised patients present unique challenges as it relates to the management of infectious diseases. This population is frequently susceptible to organisms that would otherwise be considered non-pathogenic in an immunocompetent host. Rothia is emerging as one such opportunistic infection that was previously thought to be commensal. Invasive Rothia infections have been reported in patients with hematologic malignancies, intravenous drug use, and other underlying comorbidities such as severe liver disease, alcoholism, solid tumors, end stage renal disease, and chronic lung disease [7]. Although HIV is commonly cited as a risk factor for invasive Rothia infection, to our knowledge, no previous cases of Rothia bacteremia in a patient with HIV have been reported in the literature. All previously reported invasive Rothia cases among HIV patients have been isolated from respiratory culture [1]. A recent review of Rothia bacteremia cases at a single center reported leukemia as the predominant risk factor, accounting for $61 \%$ of all cases and $89 \%$ of cases among neutropenic individuals. Among non-neutropenic patients, the most common risk factors for Rothia bacteremia were solid tumors, moderate to severe renal disease, and chronic lung disease (each accounting for $25 \%$ of non-neutropenic cases) [7]. In the case presented, risk factors for Rothia bacteremia included a diagnosis of AIDS plus non-neutropenic lymphoma with involvement of the nasopharynx and pharynx.

Given the limited ability of Rothia to consistently grow on culture media, susceptibilities are often not easily determined. Rothia is generally susceptible to penicillin, ampicillin, cefotaxime, imipenem, rifampin, and glycopeptides, but has reported resistance to aminoglycosides and macrolides [5]. However, resistance to penicillin and methicillin is increasing and can have resistance rates as high as $50 \%$ and $29 \%$ respectively, in some reports [4]. Vancomycin is an ideal choice for empiric therapy or for definitive treatment when susceptibility cannot be performed [4]. This case demonstrates a successful treatment of Rothia bacteremia utilizing the glycopeptide antibiotic, vancomycin. For invasive Rothia infections, including bacteremia in immunocompromised hosts, immediate treatment with susceptible antibiotics is warranted as Rothia can lead to significant morbidity and mortality [7].

In conclusion, we present a case of Rothia bacteremia in an immunocompromised patient with HIV and lymphoma successfully treated with a ten day course of intravenous vancomycin. Rothia susceptibilities can be 
challenging to identify due to poor growth on culture media. Vancomycin is an appropriate empiric regimen as beta-lactam resistant strains have been reported. Although rare, Rothia can cause serious opportunistic infections requiring prompt treatment with antibiotics to prevent further infectious complications.

\section{ACKNOWLEDGMENTS}

\section{Declaration of conflicting interests}

The authors declare that they have no conflict of interest.

\section{Financial disclosure}

No financial support was received.

\section{REFERENCES}

1- Maraki S, Papadakis IS. Rothia mucilaginosa pneumonia: a literature review. Infect Dis (Lond) 2015; 47(3):125-129.

2. Collins MD, Hutson RA, Baveurd V, Falsen E. Characterization of a Rothia-like organism from a mouse: description of Rothia nasimurium sp. nov. and reclassification of Stomatococcus mucilaginosus as Rothia mucilaginosa comb. nov. Int J System Evolution Microbol 2000; 50:12471251.

3. Zhang F, He S, Jin J, Dong G, Wu H. Exploring salivary microbiota in AIDS patients with different periodontal stratuses using 454 GS-FLX Titanium pyrosequencing. Front. Cell Infect Microbiol 2015; 5(55):1-10.

4. Ramanan P, Barreto JN, Osmon DR, Tosh PK. Rothia bacteremia: a 10-year experience at Mayo Clinic, Rochester, Minnesota. J of Clin Microbiol 2014; 52(9):3184-3189.

5. Von Eiff, Herrmann M, Peters G. Antimicrobial susceptibilities of Stomatococcus mucilaginosus and of Micrococcus spp. Antimicrob Agen Chemother 1995; 39(1):268-270.

6. Bruminhent J, Tokarczyk MJ, Jungkind D, DeSimone JA. Rothia mucilaginosa prosthetic device infections: a case of prosthetic valve endocarditis. J Clin Microbiol 2013;51(5):16291632.

7. Abidi MZ, Ledeboer N, Banerjee A, Hari P. Morbidity and mortality attributable to Rothia bacteremia in neutropenic and nonneutropenic patients. Diagn Microbiol Infect Dis 2016; 85(1):116-120. 